\title{
Commentary: Identification of diverse astrocyte populations and their malignant analogs
}

\author{
Chang-Hoon Cho * \\ College of Public Health, Korea University, Seoul, South Korea
}

Keywords: astrocyte, diversity, Aldh1l1, CD51, CD71, CD63

\section{A commentary on}

Identification of diverse astrocyte populations and their malignant analogs by John Lin, C. C., Yu, K., Hatcher, A., Huang, T. W., Lee, H. K., Carlson, J., et al. (2017). Nat. Neurosci. 20, 396-405. doi: 10.1038/nn.4493

Astrocytes, the most abundant type of cells in the nervous system, represent a population of complex and functionally diverse cells (Prochiantz and Mallat, 1988; Wilkin et al., 1990). It has been known since Ramon y Cajal's observation that they are heterogeneous across different brain regions as well as within the same brain regions (García-Marín et al., 2007). Astrocytes differ in their morphology (e.g., protoplasmic and fibrous), developmental origin (subventricular and hippocampal subgranular zones), gene expression profile (e.g., ion channels and transporters), astroglial coupling (e.g., gap junction proteins), and electrophysiological properties (e.g., membrane potential and potassium buffering) (Zhang and Barres, 2010; Ben Haim and Rowitch, 2017). The diverse functions of astrocytes include maintaining the bloodbrain barrier, regulating regional blood flow, providing trophic, and metabolic factors to neurons,

\section{OPEN ACCESS}

Edited by:

Detlev Boison,

Legacy Health, United States

Reviewed by:

Devin Binder

University of California, Riverside

United States

Georg Von Jonquieres, University of New South Wales,

Australia

*Correspondence:

Chang-Hoon Cho

chois007@gmail.com

Received: 29 March 2017 Accepted: 30 May 2017 Published: 13 June 2017

Citation:

Cho C-H (2017) Commentary: Identification of diverse astrocyte populations and their malignant analogs. Front. Mol. Neurosci. 10:193.

doi: 10.3389/fnmol.2017.00193 recycling neurotransmitters (uptake and release), maintaining homeostasis of neural networks, and regulating synaptogenesis and synaptic transmission (Khakh and Sofroniew, 2015). In the case of injuries (e.g., stroke and traumatic brain injury) and/or neurological and psychiatric diseases (e.g., Alzheimer's, Huntington's, Parkinson's, multiple sclerosis, and amyotrophic lateral sclerosis), astrocytes undergo diverse changes (e.g., reactive gliosis) that may be protective or causative with regard to pathologic phenotypes in a context dependent manner. Therefore, elucidating the mechanisms that expand the functional diversity of astrocytes and identifying the intrinsic and extrinsic factors for astrocytic heterogeneity have been a hot topic in glia research.

A recent report published by Drs. Benjamin Deneen and Chad Creighton at the Baylor College of Medicine has identified five distinct astrocyte subpopulations by collecting astrocytes from adult brains of ALDH1L1-eGFP mice (3-4 months old) using FACS-based techniques (John Lin et al., 2017). They selected three (CD51, CD71, and CD63) out of 81 cell surface antigens screened as markers to categorize astrocytes into distinct populations (A-E). Then, they isolated astrocytes from five different brain areas and compare the distribution of those populations. CD51, integrin alpha $\mathrm{V}$ protein (encoded by ITGAV gene), has been shown to be involved in maturation and migration of astrocytes and neuron-astrocyte interactions (Milner et al., 1999; Hermosilla et al., 2008). Increased expression of CD51 has been shown in glioblastoma (Roth et al., 2013). CD63, also called LAMP-3 and tetraspanin-30, is a lysosomal marker and it is involved in gliotransmitter release from astrocytes via $\mathrm{Ca}^{2+}$ dependent exocytosis ( $\mathrm{Li}$ et al., 2008). Increased expression of CD63 has been shown in astrocytoma (Rorive et al., 2010). CD71, transferrin receptor 1 (encoded by TFRC gene), has an increased expression in astrocytes under hypoxic condition and in glioblastoma (Yang et al., 2012; Verbovšek et al., 2014). They found that population C $\left(\mathrm{CD}^{+} 1^{+} \mathrm{CD}^{-} 1^{-} \mathrm{CD}^{-} 3^{-}\right)$ 
astrocytes, the most abundant in all brain regions examined, expressed synapse-related genes to promote the neuronal synaptogenesis and synapse function. They examined this by measuring the increased expression of presynaptic and postsynaptic markers, and increased frequency of excitatory and inhibitory postsynaptic currents in neurons when co-cultured with population $\mathrm{C}$ astrocytes. Population A $\left(\mathrm{CD} 51^{-} \mathrm{CD}^{-} 1^{-} \mathrm{CD}^{-} 3^{-}\right)$astrocytes were enriched regionspecifically in the thalamus and olfactory bulbs. They also found that population $\mathrm{C}$ is more proliferative than population A. In contrast, population A displayed more migratory potentials than population C. The distribution of five astrocyte populations from two mouse models of malignant glioma that the authors developed were examined. Populations $\mathrm{C}$ and $\mathrm{D}\left(\mathrm{CD} 1^{-} \mathrm{CD}_{71}{ }^{+} \mathrm{CD}^{-} 3^{-}\right)$were proportionally larger in population than other populations, most likely because those glioma models show severe symptoms (increased neuronal excitability and seizures) due to the increased aberrant growth of synapses. Human glioblastoma/glioma specimens were also examined for the distribution of these populations and found that population A was the majority in the limited cases shown in this study.

Many questions are raised from this interesting study. First, does this astrocytic classification cover all the astrocyte populations in the nervous system? Although Aldh1l1 has been known to be one of best astrocyte markers, other markers such as GFAP, GLAST, GLT1, GS, and AQP4 are also recognized as astrocyte-specific (Yang et al., 2011). Therefore, it may need to be examined whether isolated astrocytes from other transgenic mice expressing fluorescent proteins under other astrocyte-specific promoters could follow this classification of astrocytes. This study showed that the distribution of astrocytic subpopulations is not static. Population $\mathrm{E}\left(\mathrm{CD} 51^{-} \mathrm{CD} 71^{-} \mathrm{CD} 63^{+}\right)$is the majority in the cortex at $\mathrm{P} 28$. In contrast, this population is the minority (10\%) at the age of 3-4 months old. CD71's expression has been shown to be drastically decreased in aged mice (13 months; Duan et al., 2009). This means that CD71 may lose the capacity as a good marker for astrocyte classification beyond the young adult stages. Therefore, this classification needs to be examined as to whether it holds over the entire lifespan of animals. Otherwise, it cannot be used to classify astrocytes in old animals which

\section{REFERENCES}

Ben Haim, L., and Rowitch, D. H. (2017). Functional diversity of astrocytes in neural circuit regulation. Nat. Rev. Neurosci. 18, 31-41. doi: $10.1038 / \mathrm{nrn} .2016 .159$

Duan, W., Zhang, R., Guo, Y., Jiang, Y., Huang, Y., Jiang, H., et al. (2009). Nrf2 activity is lost in the spinal cord and its astrocytes of aged mice. In Vitro Cell. Dev. Biol. Anim. 45, 388-397. doi: 10.1007/s11626-009-9194-5

García-Marín, V., García-López, P., and Freire, M. (2007). Cajal's contributions to glia research. Trends Neurosci. 30, 479-487. doi: 10.1016/j.tins.2007 .06 .008

Hermosilla, T., Muñoz, D., Herrera-Molina, R., Valdivia, A., Muñoz, N., Nham, S. U., et al. (2008). Direct Thy-1/alphaVbeta3 integrin interaction mediates neuron to astrocyte communication. Biochim. Biophys. Acta 1783, 1111-1120. doi: 10.1016/j.bbamcr.2008.01.034 may be critical for age-related research (e.g., Alzheimer's disease). It is also possible that further classification within the same subpopulation or novel class(es) of astrocytes (with alternative surface markers) can emerge over time throughout the animals' lifespan. This might be addressed by using these three markers to sort astrocytes from the animals (e.g., CD51-eGFP mice) and examining the gene expression profiles.

Second, can this astrocyte classification be useful in injury and disease models? In these models where astrocytes undergo phenotypic changes, would these astrocyte populations play distinctive roles? Would these astrocyte populations respond differently when challenged with TGFß, FGF, or LPS either in primary culture systems or animal models in vivo? In these models and conditions, would secreted factors (e.g., GPC4/6, SPARCL1 or THBS1/2) from astrocytes differ from each population so that they have different effects on various neurons (and other astrocytes)? Would astrocyte subpopulations respond differentially to microglial secreted factors (e.g., IL-1 $\alpha$, TNF, and $\mathrm{C} 1 \mathrm{q}$ ) in inflammation or in brain disease models (Liddelow et al., 2017)?

Lastly, can this classification be traced back to the progenitors following the lineage over the embryonic period (Schitine et al., 2015)? Are distinct astrocyte populations determined at early developmental stages or they are established locally after reaching the particular brain regions (or both)?

\section{AUTHOR CONTRIBUTIONS}

The author confirms being the sole contributor of this work and approved it for publication.

\section{FUNDING}

This work was supported by Basic Science Research Program through the National Research Foundation of Korea (NRF) (NRF-2014R1A2A1A01007039).

\section{ACKNOWLEDGMENTS}

The author is pleased to acknowledge Dr. M. McCartney's comments on this manuscript.

John Lin, C. C., Yu, K., Hatcher, A., Huang, T. W., Lee, H. K., Carlson, J., et al. (2017). Identification of diverse astrocyte populations and their malignant analogs. Nat. Neurosci. 20, 396-405. doi: 10.1038/nn.4493

Khakh, B. S., and Sofroniew, M. V. (2015). Diversity of astrocyte functions and phenotypes in neural circuits. Nat. Neurosci. 18, 942-952. doi: 10.1038/nn.4043

Li, D., Ropert, N., Koulakoff, A., Giaume, C., and Oheim, M. (2008). Lysosomes are the major vesicular compartment undergoing $\mathrm{Ca}^{2+}$ regulated exocytosis from cortical astrocytes. J. Neurosci. 28, 7648-7658. doi: 10.1523/JNEUROSCI.0744-08.2008

Liddelow, S. A., Guttenplan, K. A., Clarke, L. E., Bennett, F. C., Bohlen, C. J., Schirmer, L., et al. (2017). Neurotoxic reactive astrocytes are induced by activated microglia. Nature 541, 481-487. doi: 10.1038/nature21029

Milner, R., Huang, X., Wu, J., Nishimura, S., Pytela, R., Sheppard, D., et al. (1999). Distinct roles for astrocyte alphavbeta5 and alphavbeta8 integrins in adhesion and migration. J. Cell Sci. 112, 4271-4279. 
Prochiantz, A., and Mallat, M. (1988). Astrocyte diversity. Ann. N. Y. Acad. Sci. 540, 52-63. doi: 10.1111/j.1749-6632.1988. tb27051.x

Rorive, S., Lopez, X. M., Maris, C., Trepant, A. L., Sauvage, S., Sadeghi, N., et al. (2010). TIMP-4 and CD63: new prognostic biomarkers in human astrocytomas. Mod. Pathol. 23, 1418-1428. doi: 10.1038/modpathol. 2010.136

Roth, P., Silginer, M., Goodman, S. L., Hasenbach, K., Thies, S., Maurer, G., et al. (2013). Integrin control of the transforming growth factor- $\beta$ pathway in glioblastoma. Brain 136(Pt 2), 564-576. doi: 10.1093/brain/aws351

Schitine, C., Nogaroli, L., Costa, M. R., and Hedin-Pereira, C. (2015). Astrocyte heterogeneity in the brain: from development to disease. Front. Cell. Neurosci. 9:76. doi: $10.3389 /$ fncel.2015.00076

Verbovšek, U., Motaln, H., Rotter, A., Atai, N. A., Gruden, K., Van Noorden, C. J., et al. (2014). Expression analysis of all protease genes reveals cathepsin $\mathrm{K}$ to be overexpressed in glioblastoma. PLoS ONE 9:e111819. doi: 10.1371/journal.pone. 0111819

Wilkin, G. P., Marriott, D. R., and Cholewinski, A. J. (1990). Astrocyte heterogeneity. Trends Neurosci. 13, 43-46. doi: 10.1016/0166-2236(90)90065-I
Yang, L., Fan, M., Du, F., Gong, Q., Bi, Z. G., Zhu, Z. J., et al. (2012). Hypoxic preconditioning increases iron transport rate in astrocytes. Biochim. Biophys. Acta 1822, 500-508. doi: 10.1016/j.bbadis.2011.12.004

Yang, Y., Vidensky, S., Jin, L., Jie, C., Lorenzini, I., Frankl, M., et al. (2011). Molecular comparison of GLT1+ and ALDH1L1+ astrocytes in vivo in astroglial reporter mice. Glia 59, 200-207. doi: 10.1002/glia.21089

Zhang, Y., and Barres, B. A. (2010). Astrocyte heterogeneity: an underappreciated topic in neurobiology. Curr. Opin. Neurobiol. 20, 588-594. doi: $10.1016 /$ j.conb.2010.06.005

Conflict of Interest Statement: The author declares that the research was conducted in the absence of any commercial or financial relationships that could be construed as a potential conflict of interest.

Copyright $\odot 2017$ Cho. This is an open-access article distributed under the terms of the Creative Commons Attribution License (CC BY). The use, distribution or reproduction in other forums is permitted, provided the original author(s) or licensor are credited and that the original publication in this journal is cited, in accordance with accepted academic practice. No use, distribution or reproduction is permitted which does not comply with these terms. 